\title{
STUDIES ON VALUES FOR PAIRED COMPARISON IN AHP
}

\author{
Taki Kanda \\ Bunri University of Hospitality \\ 311-1 Kashiwabarashinden \\ Sayama, Saitama 350-1336, Japan \\ kanda@bunri-c.ac.jp
}

Keywords: AHP, paired comparison, evaluation, meal intention, Kansei

\begin{abstract}
Summary: This paper is concerned with human meal preference. For evaluating human meal Kansei (Kansei means human feelings in Japanese.) a method has been proposed to give the scales for human meal feelings as subjective characteristics of food in which the units of the scales are the standard deviations of the standard normal distribution. Here in addition to the method to evaluate human meal feelings a method is discussed to evaluate individuals' meal intentions by paired comparisons, which is modified AHP(Analytic Hierarchy Process) in judging the consistency of subjects' answers and giving the scales for the answers. Combining the methods to evaluate human meal feelings and individuals' meal intentions a method is studied to evaluate individuals' meal preference. In this paper the methods to evaluate individuals' meal intentions and also individuals' meal preference are described and numerical results of the experiment on Kansei evaluation are shown..
\end{abstract}

\section{Introduction}

Modern society is an information-oriented society and the present age is also said to be "the age of Kansei ". Seeing ages in terms of the manufacturing industry in Japan the times have been changing-"the age of quantity" characterized by mass production at the period of the high growth of economy, "the age of quality” characterized by quality control, "the age of diversification” characterized by manufacture of many kinds and a small quantity of products such as CIM (Computer Integrated Manufacturing) which aims at corresponding to consumer's various needs, and “ the age of Kansei” characterized by manufacture of a product to a kind. Today which is called "the age of Kansei”, the need has been increasing to take not only the idea of product-out from companies' side but also the idea of market-in from consumers' side into consideration for planning to develop, manufacture or sell products. It is therefore effective for developing, manufacturing and selling many kinds of product to perceive consumers' needs or Kanse. Since human likes concerning foodstuff among many kinds of product are considered to be especially much influenced by Kansei, it is important to develop foodstuff and think out marketing strategies so as to fit consumers’ Kansei.

Evaluation of human likes concerning table luxuries such as coffee or beer has been studied using multivariate analysis, fuzzy theory, neural network etc., for many years. However since the present situation around food is very complicated, it is not enough to study the relation between physical properties of food and human sensations to food such as good tasting. It is necessary to study methods to evaluate human meal Kansei. Here Kansei means the function related to cognitive senses over sensations and perceptions in which human senses of value or human view points on life are included. In previous studies evaluation of human sensations to food relies mainly on flavor and texture which are known as the most important factors among various properties of food. In this study Kansei to food is evaluated using words expressing human meal Kansei..

A method has been already proposed to evaluate human meal feelings to menus on home dining tables based upon the data obtained from an experiment on Kansei evaluation. In the experiment for the method subjects are asked how much they feel that a menu is convenience-oriented one, health-oriented one and earnest-oriented one respectively and based upon subjects' answers the scales for convenience-oriented feelings, health-oriented feelings and earnest-oriented feelings to the menu are given as subjective 
characteristics of the menu. The scales obtained by this method are useful to compare subjective characteristics of food because the units of the scales are the standard deviations of the standard normal distribution ( $\sigma=1$ ) as well the scales obtained by Case of Thurston's method of paired comparisons and the total of the three scales for convenience-oriented feelings, health-oriented feelings and earnest-oriented feelings are statistically considered to be 0 . Hence the scales indicate the intention of each feeling toward the positive and negative tendencies based on zero and therefore become intelligible indications even for general consumers to express subjective characteristics of menus on home dining tables.

Here in addition to the method to evaluate human feelings to food which is already proposed, a method is studied to evaluate individuals' meal intentions. Moreover combining evaluation of human feelings to food with that of individuals' meal intentions it is studied how to evaluate individuals' preference to menus on home dining tables.

\section{Framework of Human Meal Behavior}

We must take meals to be alive and active. Regardless of our situation or interest in taking meals we could not be alive without eating. For the reason the meal behavior to conveniently take meals occurs. Besides we eat not only for the need to be alive but also for enjoyment. Enjoying meals is to search for good taste or put much importance on meal culture in taking meals. For the reason the meal behavior to earnestly take meals occurs. Moreover food has the health-related function such that if we eat this, it is good for our health or if we eat that, it is bad for our health. In this manner we care about our body when eating. For the reason the meal behavior to healthily take meals occurs.

In our towns we can find many fast food shops or convenience stores and it follows that there exit quite a few people who want to finish their meals conveniently. On the other hand when fast food shops appear firstly in Italia in 1980s, people felt that these shops will make Italian food culture ruined and in 1986 at a village BRA in Italia slow food movement arose against fast food and in 1989 slow food convivium (President: Carlo Petrini) was established aiming at preserving Italian food culture and now has more than seven hundred thousand members from all over the world. Slow food convivium is established at several places in Japan (Tokyo. Miyagi, Yamagata etc.), too. It can be naturally said that there exist many people who consider meals important and serious in terms of their attitude to life, that is take meals earnestly. In addition to human "convenience-oriented meal behavior" and "earnest-oriented meal behavior", human "health-oriented behavior" is also obvious because up to now we have been seeing various what we call "health food" or "natural food" sold and now many kinds of functional food can be found. For the above reasons in this paper letting the framework of human meal behavior be "convenience", "health", "earnest", it is studied how to evaluate human meal Kansei.

\section{Evaluation of Human Meal Intensions}

\subsection{Experiment}

An experiment on Kansei evaluation by paired comparisons was conducted to obtain the data on individuals' meal intentions letting stimuli be the words of "convenience-oriented meals", "health-oriented meals" and "earnest-oriented meals". In the experiment subjects were asked which they prefer convenience-oriented meals or health-oriented meals, health-oriented meals or earnest-oriented meals and earnest-oriented meals or convenience-oriented meals and also how much they prefer one to another.

\subsection{Consistency}

Before evaluating meal intentions, consistency of a subject's answer is judged. Table 1 shows nine answers to be chosen for the comparison of stimuli A and B and scales for the answers commonly used in AHP (Analytic Hierarchy Process). Now consider paired comparison matrix as 


$$
\mathbf{M}=\left[\begin{array}{l}
m_{11} m_{12} m_{13} \\
m_{21} m_{22} m_{23} \\
m_{31} m_{32} m_{33}
\end{array}\right]
$$

where

$$
m_{11}=m_{22}=m_{33}=1, m_{21}=1 / m_{12}, m_{31}=1 / m_{13} m_{32}=1 / m_{23} .
$$

Table 1 Scales for answers in AHP

\begin{tabular}{|l|c|c|}
\hline \multicolumn{1}{|c|}{ Answers } & A & B \\
\hline A is extremely preferred to B. & 9 & $1 / 9$ \\
\hline A is much preferred to B. & 7 & $1 / 7$ \\
\hline A is considerably preferred to B. & 5 & $1 / 5$ \\
\hline A is a little preferred to B. & 3 & $1 / 3$ \\
\hline A and B are equally preferred. & 1 & 1 \\
\hline B is a little preferred to A. & $1 / 3$ & 3 \\
\hline B is considerably preferred to A. & $1 / 5$ & 5 \\
\hline B is much preferred to A. & $1 / 7$ & 7 \\
\hline B is extremely preferred to A. & $1 / 9$ & 9 \\
\hline
\end{tabular}

Let $\lambda_{\max }$ be the maximum of eigen values, then the consistency index C.I. which shows how much a subject's answers are consistent is given by

$$
\text { C.I. }=\frac{\lambda_{\max }-n}{n-1}
$$

where $n$ is the degree of paired comparison matrix. Generally if $C . I . \leq 0.1$, it is empirically judged that the answers are consistent. To obtain $\lambda_{\max }$, it is needed to solve the following algebraic equation which is the egein equation of the paired comparison matrix of (1).

$$
-\lambda^{3}+3 \lambda^{2}+\frac{m_{13}}{m_{12} m_{23}}+\frac{m_{12} m_{23}}{m_{13}}-2=0
$$

Although algebraic equations of higher degree are able to be numerically solved by numerical methods such as the DKA method (the Durand-Kerner-Aberth method) or modified .DKA method, in order to make the judgment on the consistency of a subject's answers simple so that even general consumers at home can easily judge the consistency, a criterion of the consistency is considered without solving the algebraic equation of (3). To do this, consider the function

$$
f(\lambda)=-\lambda^{3}+3 \lambda^{2}+\frac{m_{13}}{m_{12} m_{23}}+\frac{m_{12} m_{23}}{m_{13}}-2
$$

Taking the term without variable $\lambda$ in (4) let

$$
C=\frac{m_{13}}{m_{12} m_{23}}+\frac{m_{12} m_{23}}{m_{13}}-2
$$

where $C$ is the intercept to ordinate in the graphs of $f(\lambda)$.

Now when the consistency index, C.I. $=0$ the subject's answers are perfectly consistent and we have $\lambda_{\max }$ and $C=0.000$ as shown in Figure 1.Figure 2 shows the graph of $f(\lambda)$ in the case where 
C.I. $=0.1$ which is the threshold of consistency. In this case we have $\lambda_{\max }=3.2$ and $C=2.048$ as shown in the figure.

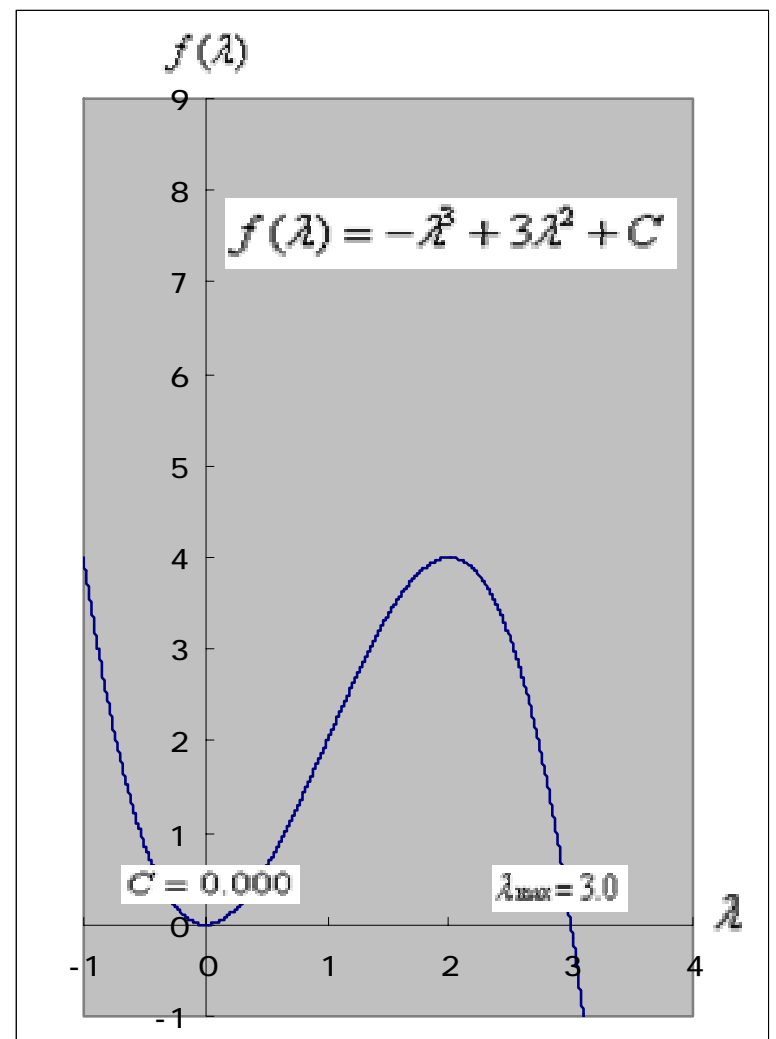

Figure 1 Graph of $f(\lambda)$ in the case where C.I. $=0$

Figure 3 shows the graph of $f(\lambda)$ in the case where C.I. $=0.15$ which means that the subject's answers are inconsistency. In this case we have $\lambda_{\max }=3.3$ and $C=3.267$ as shown in the figure. In terms of those figures it is stated that the criteria for the consistency of a subject's answers becomes $\lambda_{\max } \leq 3.2$ and it follows $C \leq 2.048$.

Now in order to simplify the judgment on the consistency, simplified consistency index S.C.I. is defined by

$$
\text { S.C.I. }=\frac{C}{2.048}
$$

and then

$$
\text { S.C.I. } \leq 1
$$

turns out to be the criterion for the consistency of a subject's answers. Therefore instead of obtaining $\lambda$ by solving the algebraic equation of (3) it is able to be judged by simple arithmetical operations of (5) and (6) that if (7) is satisfied, the subject's answers are consistent. 


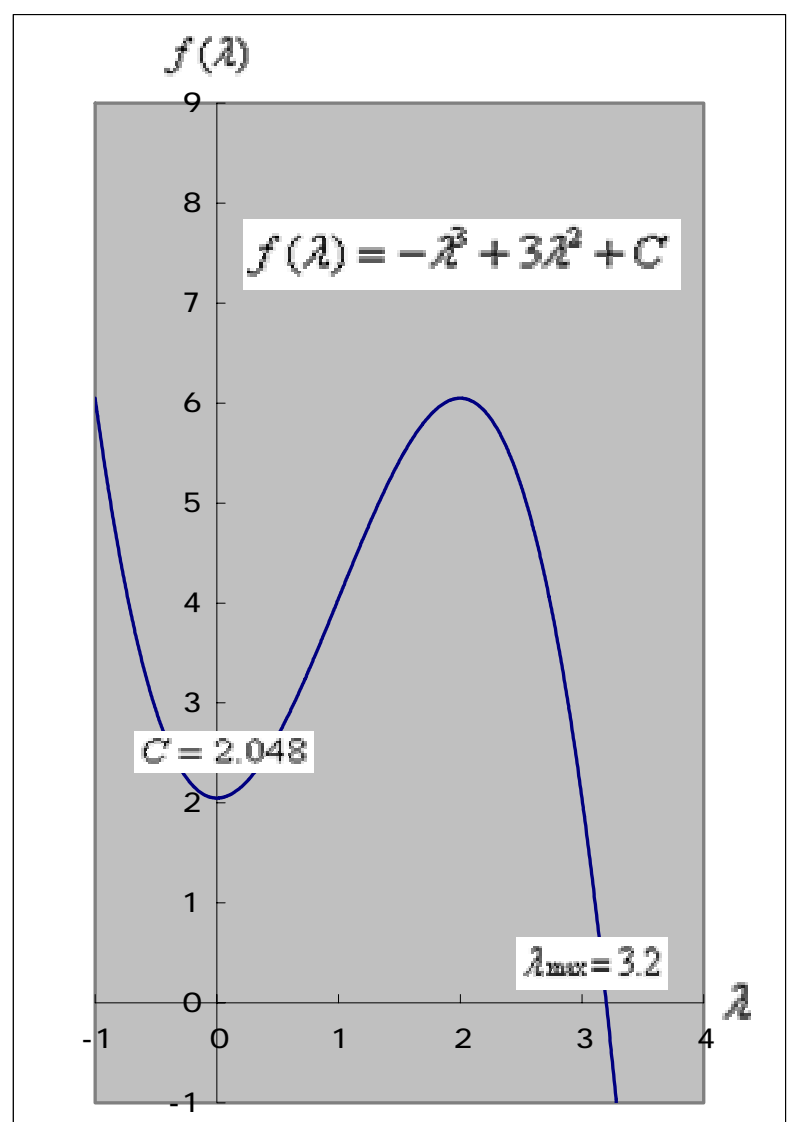

Figure 2 Graph of $f(\lambda)$ in the case where C.I. $=0.1$

\subsection{Meal Intentions}

There are many methods of paired comparisons which have been proposed by Thurston, Scheffe etc. since Psychophysics had been proposed in nineteenth century, and AHP is one of the methods the essence of which is the paired comparison. The values to give to the answers of paired comparisons vary with method and should be considered according to the purpose to use paired comparisons. For example, in Guttman's method which quantifies paired comparisons by eigenvalue problems as well as AHP proposed by Saaty the values to the answers are 1 or 0 and in Fuzzy AHP they are fuzzy numbers. In this study order statistics defied based upon the standard normal distribution are used to make the units of the scales for meal intentions equivalent to the standard deviations of the standard normal deviation. For the comparison of stimuli A and B, the scales by order statistics are given in accordance with subjects' answers as shown in Table 2. 


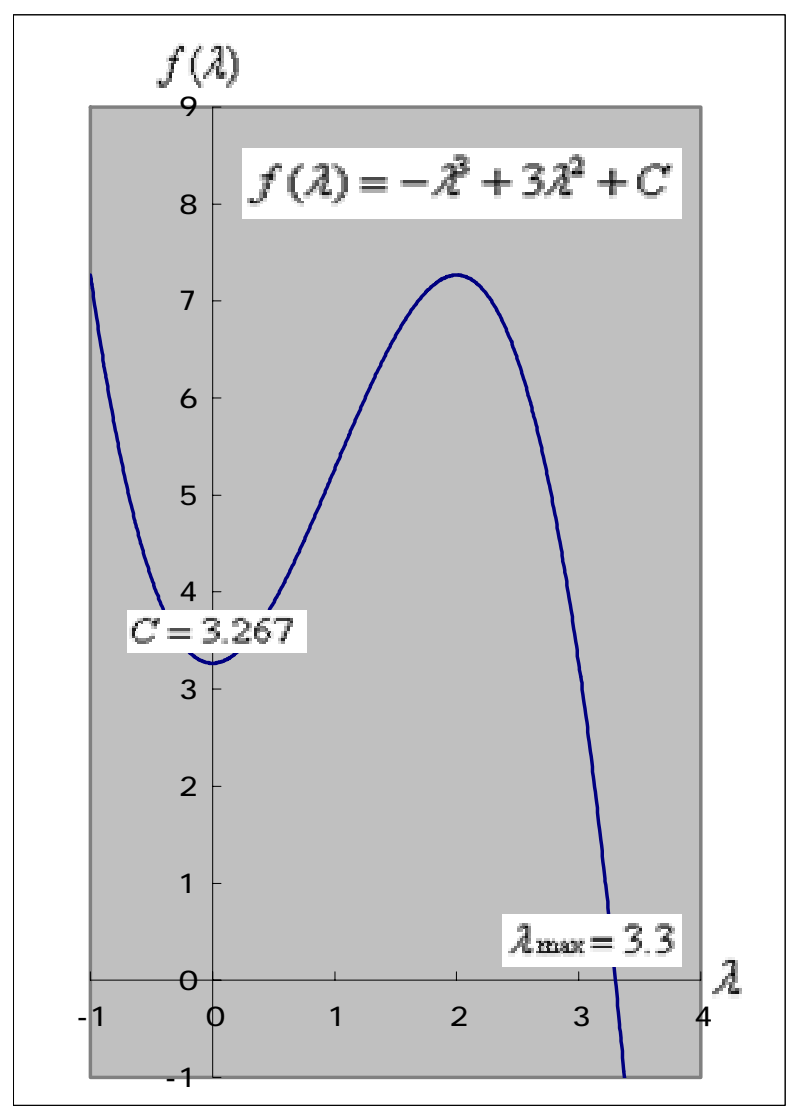

Figure 3 Graph of $f(\lambda)$ in the case where C.I. $=0.15$

Table 2 Scales for answers by order statistics

\begin{tabular}{|l|c|c|}
\hline \multicolumn{1}{|c|}{ Answers } & A & B \\
\hline A is extremely preferred to B. & 1.485 & -1.485 \\
\hline A is much preferred to B. & 0.932 & -0.932 \\
\hline A is considerably preferred to B. & 0.572 & -0.572 \\
\hline A is a little preferred to B. & 0.275 & -0.275 \\
\hline A and B are equally preferred. & 0.000 & 0.000 \\
\hline B is a little preferred to A. & -0.275 & 0.275 \\
\hline B is considerably preferred to A. & -0.572 & 0.572 \\
\hline B is much preferred to A. & -0.932 & 0.932 \\
\hline B is extremely preferred to A. & -1.485 & 1.485 \\
\hline
\end{tabular}

Now consider the following paired comparison matrix each component of which is order statistics [20].

$$
\mathbf{N}=\left[\begin{array}{lll}
n_{11} & n_{12} & n_{13} \\
n_{21} & n_{22} & n_{23} \\
n_{31} & n_{32} & n_{33}
\end{array}\right]
$$

where 


$$
n_{11}=n_{22}=n_{33}=0, n_{21}=-n_{12}, n_{31}=-n_{13}, n_{32}=-n_{23} \text {. }
$$

Since the matrix (8) is not the positive reciprocal matrix, the geometric mean method which is considered a convenient method of the eigenvvalue method is not able to be used to obtain the weights to alternatives. Therefore let $I_{c}, I_{h}, I_{e}$ be the scales for convenience-oriented meal intentions, health-oriented meal intentions and earnest-oriented meal intentions of a subject respectively and they are given as the arithmetic average of each row of (8), that is

$$
\begin{aligned}
& I_{c}=\frac{n_{11}+n_{12}+n_{13}}{3} \\
& I_{h}=\frac{n_{21}+n_{22}+n_{23}}{3} \\
& I_{e}=\frac{n_{31}+n_{32}+n_{33}}{3} .
\end{aligned}
$$

Since $I_{c}, I_{h}, I_{e}$ are the average of the values of order statistics, the units of them are able to be considered the standard deviations of the standard normal distribution.

\subsection{Results of Experiment}

An experiment on Kansei evaluation to know human meal intentions was conducted, the subjects of which were 7 University students. The answers of 7 subjects and simplified consistency index S.C.I. calculated from the answers are as follows.

Subject No.1:

In the comparison of convenience-oriented meals and health-oriented meals, convenience-oriented meals were much preferred to health-oriented meals.

In the comparison of health-oriented meals and earnest-oriented meals, health-oriented meals were considerably preferred to earnest-oriented meals.

In the comparison of earnest-oriented meals and convenience-oriented meals, convenience-oriented meals were extremely preferred to earnest-oriented meals.

For the results simplified consistency index S.C.I. is calculated to be 0.399 which is not more than 1

\begin{tabular}{|c|c|c|c|c|}
\hline & $\mathrm{C}$ & $\mathrm{H}$ & $\mathrm{E}$ & Average \\
\hline $\mathrm{C}$ & 0.000 & 0.932 & 1.485 & 0.806 \\
\hline $\mathrm{H}$ & -0.932 & 0.000 & 0.572 & -0.120 \\
\hline$E$ & -1.485 & -0.572 & 0.000 & -0.686 \\
\hline \multicolumn{3}{|c|}{ S.C.I. $=0.399$} & Total & 0.000 \\
\hline
\end{tabular}
thus it is decided that the answers of subject No.1 are consistent. The scales for the three intentions are obtained as shown in Table 3.

Table 3 Scales for meal intentions of subject No.1

C: Convenience H: Health E: Earnest

S.C.I.: Simplified Consistency Index

Subject No.2:

In the comparison of convenience-oriented meals and health-oriented meals, convenience-oriented meals were much preferred to health-oriented meals.

In the comparison of health-oriented meals and earnest-oriented meals, earnest-oriented meals were a little preferred to health-oriented meals.

In the comparison of earnest-oriented meals and convenience-oriented meals, convenience-oriented meals were considerably preferred to earnest-oriented meals. 
For the results simplified consistency index S.C.I. is calculated to be 0.082 which is not more than 1 thus it is decided that the answers of subject No.2 are consistent. The scales for the three intentions are obtained as shown in Table 4.

Table 4 Scales for meal intentions of subject No.2

\begin{tabular}{|c|c|c|c|c|}
\hline & $\mathrm{C}$ & $\mathrm{H}$ & $\mathrm{E}$ & Average \\
\hline $\mathrm{C}$ & 0.000 & 0.932 & 0.572 & 0.501 \\
\hline $\mathrm{H}$ & -0.932 & 0.000 & -0.275 & -0.402 \\
\hline $\mathrm{E}$ & -0.572 & 0.275 & 0.000 & -0.099 \\
\hline \multicolumn{3}{|c|}{ S.C.I. $=0.082$} & Total & 0.000 \\
\hline
\end{tabular}

$\mathrm{C}$ : Convenience $\mathrm{H}$ : Health $\mathrm{E}$ : Earnest

S.C.I.: Simplified Consistency Index

Subject No.3:

In the comparison of convenience-oriented meals and health-oriented meals, health-oriented meals were considerably preferred to convenience-oriented meals.

In the comparison of health-oriented meals and earnest-oriented meals, health-oriented meals were considerably preferred to earnest-oriented meals.

In the comparison of earnest-oriented meals and convenience-oriented meals, convenience-oriented meals were a little preferred to earnest-oriented meals.

For the results simplified consistency index S.C.I. is calculated to be 0.244 which is not more than 1 thus it is decided that the answers of subject No.3 are consistent. The scales for the three intentions are obtained as shown in Table 5.

Table 5 Scales for meal intentions of subject No.3

\begin{tabular}{|c|c|c|c|r|}
\cline { 2 - 5 } \multicolumn{1}{c|}{} & $\mathrm{C}$ & $\mathrm{H}$ & $\mathrm{E}$ & Average \\
\hline $\mathrm{C}$ & 0.000 & -0.572 & 0.275 & -0.099 \\
\hline $\mathrm{H}$ & 0.572 & 0.000 & 0.572 & 0.381 \\
\hline $\mathrm{E}$ & -0.275 & -0.572 & 0.000 & -0.282 \\
\hline \multicolumn{3}{c}{ S.C.I. $=0.244$} & Total & 0.000 \\
\hline
\end{tabular}

C: Convenience H: Health E: Earnest

S.C.I.: Simplified Consistency Index

Subjects No.4:

In the comparison of convenience-oriented meals and health-oriented meals, health-oriented meals were extremely preferred to convenience-oriented meals.

In the comparison of health-oriented meals and earnest-oriented meals, health-oriented meals were considerably preferred to earnest-oriented meals.

In the comparison of earnest-oriented meals and convenience-oriented meals, earnest-oriented meals were much preferred to convenience-oriented meals.

For the results simplified consistency index S.C.I. is calculated to be 0.399 which is not more than 1 thus it is decided that the answers of subject No.4 are consistent. The scales for the three intentions are obtained as shown in Table 6.

Table 6 Scales for meal intentions of subject No.4

\begin{tabular}{|c|c|c|c|c|}
\cline { 2 - 5 } \multicolumn{1}{c|}{} & $\mathrm{C}$ & $\mathrm{H}$ & $\mathrm{E}$ & Average \\
\hline $\mathrm{C}$ & 0.000 & -1.485 & -0.923 & -0.806 \\
\hline $\mathrm{H}$ & 1.485 & 0.000 & 0.572 & 0.686 \\
\hline $\mathrm{E}$ & 0.923 & -0.572 & 0.000 & 0.120 \\
\hline \multicolumn{3}{c}{ S.C.I. $=0.399$} & Total & 0.000 \\
\hline
\end{tabular}

C: Convenience H: Health E: Earnest

S.C.I.: Simplified Consistency Index

Subject No.5: 
In the comparison of convenience-oriented meals and health-oriented meals, health-oriented meals were considerably preferred to convenience-oriented meals.

In the comparison of health-oriented meals and earnest-oriented meals, earnest-oriented meals were a little preferred to convenience-oriented meals.

In the comparison of earnest-oriented meals and convenience-oriented meals, earnest-oriented meals were extremely preferred to convenience-oriented meals.

For the results simplified consistency index S.C.I. is calculated to be 0.016 which is not more than 1 thus it is decided that the answers of subject No.5 are consistent. The scales for the three intentions are obtained as shown in Table 7.

Table 7 Scales for meal intentions of subject No.5

\begin{tabular}{|c|c|c|c|c|}
\hline & $\mathrm{C}$ & $\mathrm{H}$ & $\mathrm{E}$ & Average \\
\hline $\mathrm{C}$ & 0.000 & -0.572 & -1.485 & -0.686 \\
\hline $\mathrm{H}$ & 0.572 & 0.000 & -0.275 & 0.099 \\
\hline$E$ & 1.485 & 0.275 & 0.000 & 0.587 \\
\hline \multicolumn{3}{|c|}{ S.C.I. $=0.016$} & Total & 0.000 \\
\hline
\end{tabular}

C: Convenience H: Health E: Earnest

S.C.I.: Simplified Consistency Index

\section{Evaluation of Meal Preference}

\subsection{Definition of Scales}

To determine the scales for meal preference the idea of AHP is applied letting the elements of criteria be subjective characteristics of food, that is human convenience-oriented feelings, health-oriented feelings and earnest-oriented feelings to food and alternatives be menus on home dining tables. The original AHP of Saaty is called a relative measurement in which paired comparisons are used to evaluate the elements of criteria with respect to each alternative. In this study the elements of criteria are human convenience-oriented feelings, health-oriented feelings and earnest-oriented feelings and the sales for those feelings with respect to each menu are obtained using the already proposed method which is based upon human evaluation by 5 grades as described in 1 . It is therefore able to be said that the method discussing here is a kind of modified absolute measurement in AHP. Seeing this study in terms of AHP first hierarchy level is "selection of meals" ( Level 1 ), second hierarchy level is "subjective characteristics of food” ( Level 2 ) and third hierarchy level is "menus on home dining tables” ( Level 3 ) as shown in Figure 4.

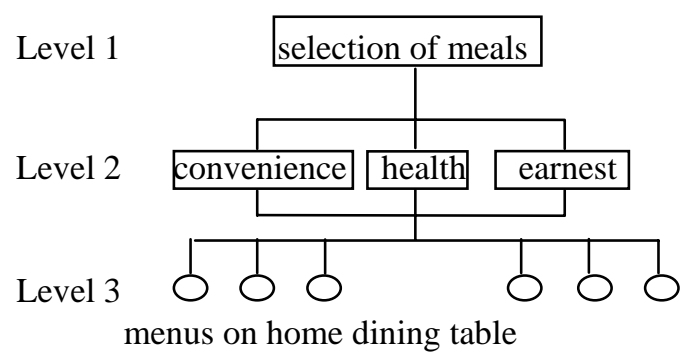

Figure 4 A hierarchy for preference of menus on home dining tables

In accordance with Figure 4 the scales for meal preference of a subject to a menu are obtained as follows. Let $I C_{j}, I H_{j}, I E_{j}$ be the scales for convenience-oriented meal Intentions, health-oriented meal intentions and earnest-oriented meal intentions of subject $j$ respectively and $F C_{i}, F H_{i}, F E_{i}$ be the scales for convenience-oriented feelings, health-oriented feelings and earnest-oriented feelings to menu $i$ respectively. Then the scales for meal preference of subject $j$ to menu $i$ are given by 
( i ) if $I C_{j} \times F C_{j}+I H_{j} \times F H_{j}+I E_{j} \times F E_{j} \geq 0$,

$$
P_{i j}=\sqrt{I C_{j} \times F C_{i}+I H_{j} \times F H_{i}+I E_{j} \times F E_{i}}
$$

(ii) if $I C_{j} \times F C_{j}+I H_{j} \times F H_{j}+I E_{j} \times F E_{j}<0$,

$$
P_{i j}=-\sqrt{-\left(I C_{j} \times F C_{1}+I H_{j} \times F H_{i}+I E_{j} \times F E_{l}\right)} .
$$

Since $P_{i j}$ are calculated as the square root of the sum of products of the scale for human meal feelings and that of individuals' meal intentions and the units of both scales for human meal feelings and individuals' meal intentions are the standard deviations of the standard normal distribution, the units of $P_{i j}$ turns out to be the standard deviations of the standard normal distribution. Besides, if the given scales are positive, they are considered to indicate the intention of preference to the menu and if the given scales are negative, they are considered to indicate the intention of non-preference to the menu.

\section{Concluding Remarks}

In this paper we were concerned with human meal Kansei . First, the framework of human meal behaviors was considered and we have come to the conclusion that human meal behaviors are broadly divided into a convenience-oriented meal behavior, a health-oriented meal behavior and an earnest-oriented meal behavior, and based upon the frame work a method was discussed to evaluate individuals' preference to menus on home

dining tables applying the idea of AHP..

In order to know our meal preference a method was studied to evaluate individuals' convenience-oriented meal intentions, health-oriented meal intentions and earnest-oriented meal intentions, and combining this with the already proposed method to evaluate human convenience-oriented feelings, health-oriented feelings and earnest-oriented feelings to menus on home dining tables a method was studied to evaluate individuals' meal preference in which the scales for preference are given so that the units can be considered the standard deviations of the standard normal distribution.

The methods discussed here might be useful to know our meal Kasei even for general consumers and expected to be applied to foodstuff development, production or marketing

\section{References}

Satty, T. L. (1977), “A Scaling Method for Priorities in Hierarchical Structures” Journal of Mathematical Psychology; 15, 234-28

Yamauchi; Z. (1972) STATISTICAL TABLES and Formulas with Computer Applications JSA-1972; JAPANESE STANDARDS ASSOCIATION, 33-49

Kinoshita, E. and Nakanishi, M. (1999), "Proposal of New AHP Model in Light of Dominant Relationship among Alternatives”, Journal of the Operations Research Society of Japan; 42(2), 180-197.

Kanda, T. (2004), "Evaluation of Human Meal Preference Based upon Human Meal Feelings and Intentions” International Journal of Kansei: Engineering, 4(2), 9-18 . 